\title{
Modelling the effects of social networks in interventions aimed at reducing meat consumption using a social simulation
}

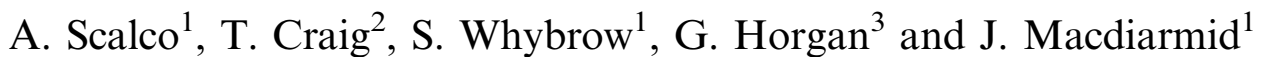 \\ ${ }^{1}$ Life Course \& Population Health, The Rowett Institute, University of Aberdeen, Aberdeen, AB25 2ZD, \\ ${ }^{2}$ Social, Economic \& Geographical Sciences Research Group, The James Hutton Institute, Aberdeen, AB15 8QH and \\ ${ }^{3}$ Biomathematics \& Statistics Scotland, Aberdeen, AB25 2ZD
}

High levels of meat consumption are a problem for both the environment and health. Livestock production is associated with high levels of greenhouse gas emissions and there are concerns about an increased health risk from high intakes of red and processed meat. However, the challenge is how to reduce meat consumption when meat-based diets are the norm. The influence of social networks on food choices is important for changing norms but rarely considered in nutrition-based interventions. This study aimed to model the effect of social networks in workplace interventions to reduce meat consumption.

This work developed a social simulation of British consumers, using agent-based modelling, to explore the impact of strategies to reduce meat consumption through networks ${ }^{(1,2)}$. Agents in the model represent consumers influenced by prices and by their personal views about the impact of meat on the environment, health, and animal welfare. Each agent had two social networks (one at work and another at home), which could influence their decision about eating a meal with or without meat. Agents' characteristics were defined using data from the British Attitudes Survey ${ }^{(3)}$. Data from the National Diet and Nutrition Survey (2008/9 to 2013/14) ${ }^{(4,5)}$ were used to empirically ground the model and the outputs included the average weekly consumption of meat and the likelihood of eating meat. A series of experiments were run simulating different real-world interventions: increasing the price of meat (5/10/20\%); providing norm-based messages in the workplace with three different messages about meat (environment, health, or animal welfare); altering frequency of messages; target specific groups of the population based on their concerns.

The model reproduced observed consumption patterns from the past years, which gave reliability of the model. A $20 \%$ price increase had the greatest impact on lowering consumption. A price increase of $5 \%$ on meat products had a greater effect on reducing meat consumption among workers than environmental and animal welfare messages but not greater than health messages. The simulations showed exposure to norm-based messaging interventions in the workplace could affect both workers and spillover to household members in a positive manner via social influences within and across networks. Repeating messages had a greater impact on changing behaviours compared to a one-off intervention over the same time period. An unintended consequence emerged with meat consumption increasing over time when the targeted groups comprised those with the greatest concerns.

This study provides insights into the effectiveness of networks and social influence to shift the population towards healthier and sustainable diets. Using a modelling approach provides a way to identify potential outcomes and unintended consequences of interventions, which is a cost effective and efficient way to explore an intervention prior to implementation.

1. Scalco A et al. (2019) J. Artificial Societies and Social Simulations, 22, 4.

2. CoMSES Computational Model Library [Available at: https://www.comses.net/codebases/e70769cd-06fa-4339-b9b4-6d4679701ce4/releases/1.0.0/]

3. NatCen Social Research (2016) UK

4. Bates B, et al. (2014) Public Health England. [Available at: https://www.gov.uk/government/statistics/national-diet-and-nutrition-survey-resultsfrom-years-1-to-4-combined-of-the-rolling-programme-for-2008-and-2009-to-2011-and-2012]

5. Bates B, et al. (2016) Public Health England. [Available at: https:/www.gov.uk/government/statistics/ndns-results-from-years-5-and-6-combined] 